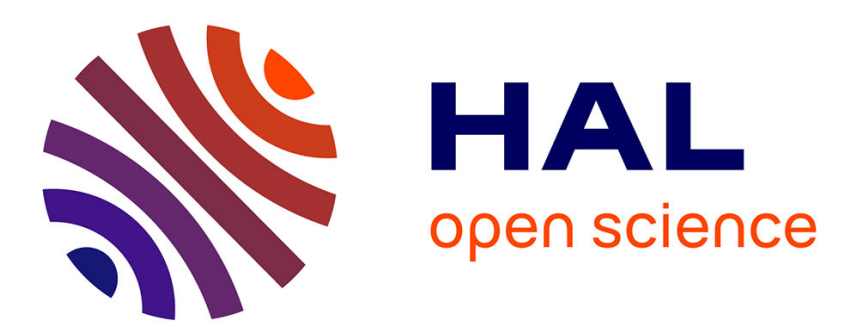

\title{
La construction locale de l'offre d'une prestation comme production de l'action sociale: le cas du Fonds d'Urgence Sociale
}

\author{
Gilles Frigoli
}

\section{- To cite this version:}

Gilles Frigoli. La construction locale de l'offre d'une prestation comme production de l'action sociale: le cas du Fonds d'Urgence Sociale. Revue française des affaires sociales, 2001, 1, pp.185-197. halshs00098259

\section{HAL Id: halshs-00098259 \\ https://shs.hal.science/halshs-00098259}

Submitted on 25 Oct 2006

HAL is a multi-disciplinary open access archive for the deposit and dissemination of scientific research documents, whether they are published or not. The documents may come from teaching and research institutions in France or abroad, or from public or private research centers.
L'archive ouverte pluridisciplinaire HAL, est destinée au dépôt et à la diffusion de documents scientifiques de niveau recherche, publiés ou non, émanant des établissements d'enseignement et de recherche français ou étrangers, des laboratoires publics ou privés. 


\title{
La construction locale de l'offre d'une prestation comme production de l'action sociale : le cas du Fonds d'Urgence Sociale
}

\author{
Gilles Frigoli*
}

Conçue initialement comme une réponse politique nationale à des mouvements de chômeurs largement médiatisés, la mise en place du FUS a rapidement pris la forme d'une opération de sélection des publics prioritaires de l'action sociale, en vue de l'octroi d'une aide financière dont les règles d'attribution furent largement laissées à l'appréciation des autorités départementales. Celles-ci furent alors amenées à se doter de critères afin d'assurer le ciblage des publics du FUS. En proie à cet égard à une relative incertitude et sous le poids des contraintes liées à la constitution d'un partenariat local, les instances chargées du pilotage du dispositif dans le département où nous avons enquêté ${ }^{1}$ furent conduites à faire reposer en partie cette sélection sur l'usager lui-même, sur sa capacité à se reconnaître comme cible et à motiver sa demande, ainsi que sur sa détermination à faire valoir la pertinence de cette auto-désignation.

Partant de ce constat, on propose de montrer comment, à partir de ce choix logistique, le dispositif a trouvé un public. Plus précisément, on s'attache à comprendre comment se rencontrent offre et demande de prestation lorsque, comme ce fut le cas ici, la rationalité de l'usager est mise à contribution pour réduire l'incertitude sur la définition de la cible visée.

\section{Un questionnement sur les logiques de sélection des publics prioritaires de l'action sociale}

Le FUS peut être appréhendé comme une expérience massive et ponctuelle de ciblage de populations qui, alors qu'elles font l'objet d'une attention publique sans cesse renouvelée, ne se laissent que difficilement enfermer dans une définition normative. Incontestablement, le triptyque "pauvreté-précarité-exclusion", mobilisé sur la scène politico-médiatique pour évoquer le sort des victimes de la "nouvelle question sociale", fonctionne socialement. Mais tout se passe comme si l'efficacité sociale de ces notions était à la mesure de leur imprécision².

\footnotetext{
- Publié dans : Frigoli G., "La construction locale de l'offre d'une prestation comme production de l'action sociale : le cas du Fonds d’Urgence Sociale", Revue Française des Affaires Sociales, n 1 , janvier-mars 2001, pp.133-155.

* SOLIIS-URMIS (ESA CNRS 7032), Université de Nice-Sophia Antipolis

${ }^{1}$ Dans le cadre du programme d'études "Mieux connaître les publics, les problèmes et les pratiques révélés par la mise en place des Fonds d'urgence sociale" (Ministère de l'Emploi et de la Solidarité/DAS), notre enquête a porté sur la mise en place du FUS dans les Alpes-Maritimes. L'étude a été réalisée avec la collaboration de Christian Rinaudo (SOLIIS-URMIS).

${ }^{2}$ On pourrait, pour s'en convaincre, mettre en parallèle leur fort pouvoir d'évocation et les limites que connaissent les diverses méthodes de construction statistique des catégories de population correspondantes. Si l'on met en effet de côté les approches qui se cantonnent au recensement des publics faisant l'objet d'une prise en charge sociale, dont la portée analytique est pour le moins limitée, on ne peut que constater les difficultés qu'engendre toute classification des personnes défavorisés fondée sur l'attribution de traits individuels caractéristiques, difficultés que la sophistication croissante des critères et des modes de calcul ne parvient que partiellement à combler. Ainsi, les spécialistes se voient-ils aujourd'hui conduits à emprunter au vocabulaire médical pour rendre compte de situations considérées comme complexes et évolutives — on parlera alors de «symptômes » et de «facteurs aggravants »; à associer les populations à la construction des critères de définition — on pense au principe de «pauvreté subjective »; à finalement consacrer la pertinence d'une approche en termes de risques, au prix de la création
} 
Or, c'est justement en laissant largement ouvert le choix du mode de constitution des catégories de populations visées par le FUS que l'administration centrale invita les services déconcentrés à opérer une sélection parmi cet ensemble aux contours imprécis que constitue le groupe des "personnes en difficulté". Et, au moins dans un premier temps, les autorités locales ne purent s'appuyer que sur une série d'énoncés politiques volontaristes, puisant en partie au registre de l'émotion en réaction à des mouvements de revendication et supposant leur intelligibilité immédiate. Ainsi, les Préfectures et les DDASS furent-elles exhortées à apporter une aide financière aux personnes et aux ménages touchés par la détresse sociale, à mettre un terme aux situations d'urgence sociale, et, par là, à donner un contenu à ces notions qui furent sans doute convoquées pour l'efficacité sociale que garantit leur expressivité. Mais il restait, à travers l'organisation locale des conditions de mise en oeuvre de cette discrimination positive, à en tirer des principes de sélection opératoires.

Dans le département sur lequel a porté notre enquête, les instances de pilotage du dispositif optèrent pour une définition non restrictive du public concerné par le FUS. Et, pour des raisons qu'on évoquera, elles ne purent s'appuyer sur le concours officiel des travailleurs sociaux pour en affiner le contenu. Les personnes connaissant des difficultés furent invitées par voie de presse à retirer un dossier de demande en mairie et à y exposer les raisons qui leur semblaient de nature à justifier l'attribution d'une aide financière, le choix des éléments de preuve à l'appui de cette demande étant laissé dans une large mesure à leur apréciation. C'est donc à l'usager lui-même que revint en partie la charge de définir la cible du dispositif, en motivant par écrit le bien-fondé de cette définition auprés d'une commission d'attribution centralisée fondant ses décisions sur l'établissement d'une "jurisprudence" ad hoc. C'est dire qu'à l'occasion de la construction locale de l'offre de cette prestation, c'est le choix de la légitimité accordée à la parole de l'usager qui prévalut sur celui de la rationalisation des procédures de sélection. Et c'est cette légitimité qui fut officiellement appelée à combler l'espace d'interprétation qui sépare un discours politique national pariant sur l'évidence de significations socialement partagées, de citoyens-usagers qui furent en l'occurence appelés à se reconnaître dans la formulation de la réponse publique correspondante.

Les questions que soulève ce mode de sélection des personnes qu'il faut aider en priorité sont nombreuses. Pour certains, pointe ici le spectre de l'assistance, version charité, et son cortège de représentations négatives : absence d'égalité de traitement, "prime à la débrouillardise", subjectivité des critères d'attribution, etc. Même si ce débat ne doit pas être écarté, c'est sur un autre plan, plus empirique, qu'on voudrait conduire l'analyse, en s'interrogeant sur le destin de ce principe d'action initial au cours des semaines puis des mois qui suivirent l'installation du FUS dans le département. Qu'est-il advenu de la légitimité accordée à l'usager lorsque les premiers dossiers de demande sont arrivés en Préfecture et qu'il a fallu les trier ? Lorsque des intervenants sociaux ont eu connaissance de la mise en place de cette aide accordée par le gouvernement et ne nécessitant ni instruction par un travailleur social, ni pièces justificatives spécifiées?

continue de nouvelles catégories, sorte de fuite en avant classificatoire dont la figure du «working poor», objet actuel de préoccupations grandissantes, offre une illustration. 
En répondant à ces questions, on espère apporter une contribution à une réflexion plus générale. Celle-ci porte sur le mode de désignation des publics prioritaires de l'aide et de l'action sociales. Trois modes opératoires peuvent être distingués qui s'appuient sur des légitimités différentes : la référence, comme ce fut le cas pour le FUS dans le département étudié, à un usager doté de capacités d'autonomie qui justifient qu'on s'en remette dans une large mesure à ce dernier pour avancer, de manière performative, une définition de la population concernée ; l'élaboration a priori de règles et de critères de sélection permettant une catégorisation «étanche » puis un traitement automatique des situations ; le recours à la technicité professionnelle d'intervenants sociaux administrativement mandatés pour adapter, face au demandeur potentiel et en vertu de la compétence qui leur est prêtée, des principes généraux à des situations particulières. On tentera de montrer que ce que met en jeu la première de ces trois voies, c'est moins le risque d'opérer une sélection injuste ou non pertinente dans ses choix, que celui de priver les acteurs, du côté de l'offre comme de la demande, de la possibilité de se réunir autour du sens de la démarche d'aide.

\section{Les enjeux de la construction de l'offre de prestation à l'épreuve du local}

Avant de voir comment s'est effectivement déployée l'offre de prestation en fonction des principes adoptés localement qu'on vient d'évoquer, il faut rappeler que des contraintes fortes pesaient sur les choix des autorités locales. Celles-ci ne disposèrent que de peu de temps pour organiser la mise en place du FUS et, de plus, il leur fallut compter avec les attentes, parfois les réticences, de certains de leurs partenaires institutionnels. Il convient donc de replacer l'analyse dans le contexte des négociations qui ont conditionné la construction institutionnelle de l'accès au FUS.

\section{Un pragmatisme obligé dans la gestion des contraintes liées au partenariat}

Dés réception de la circulaire d'application relative au FUS, c'est essentiellement à la DDASS que revint la charge de concevoir la mise à disposition de la population de cette prestation nouvelle puis de formaliser une définition des publics concernés par le dispositif.

Le premier volet engageait deux types de démarche : la recherche d'institutions partenaires susceptibles de contribuer à la mise en place du dispositif ainsi que l'élaboration d'un schéma départemental fixant les modalités de la rencontre entre offre et demande de prestation. La marge de maneuvre de l'administration sociale était limitée, d'un côté par le fait qu'elle ne dispose pas d'un réseau de professionnels de proximité capable de couvrir l'ensemble du territoire, de l'autre par la réticence du Conseil Général qui se montrait peu enclin à s'engager sur une mobilisation immédiate et systématique de ses travailleurs sociaux. Devant la réserve affichée à ce sujet par les autres organismes dotés de points d'accueil des publics, la décision fut prise de solliciter le concours de l'ensemble des mairies du département, constituées en guichets de distribution des formulaires de demande. Cette dernière devrait alors être effectuée sans instruction préalable par un tiers, donc être assurée par le demandeur lui-même, avant d'être examinée par une commission centralisée (MUS) constituée de membres d'organismes gestionnaires de fonds sociaux. Bien que largement contraint par l'absence de ressources professionnelles disponibles 
pour l'action et la nécessité de disposer d'une logistique immédiatement opérationnelle, ce choix trouvera sa justification officielle dans l'appel à la vocation citoyenne des mairies, le relatif anonymat qu'en tant que lieu d'accueil celles-ci garantissent à la démarche et la simplicité de la procédure requise, à condition que l'information soit diffusée de manière suffisamment large. A cette fin, la création du dispositif sera annoncée par voie de presse et le message relayé par affichage du formulaire au sein des lieux d'accueils dont disposent les institutions composant la commission.

On notera cependant que le choix de ce principe d'action servait également implicitement l'indétermination qui caractérisait le ciblage de la population au tout début du dispositif en permettant, dans l'attente d'une hypothétique clarification, de ne privilégier ni d'écarter aucune catégorie de publics a priori. En effet, pour plusieurs raisons, l'élaboration d'une définition opératoire des publics visés s'apparentait plus à un exercice de production sous contrainte d'une cible et d'objectifs à atteindre qu'à la stricte application déconcentrée de directives techniques ${ }^{3}$.

Dans ce contexte d'indétermination relative, le ciblage à usage interne, destiné aux membres de la commission, prendra la forme d'une "doctrine empirique" appelée à s'affiner progressivement. Sur les deux supports officiels de diffusion de l'information, à savoir la presse locale et le formulaire de demande, la définition de la cible à destination de la population combinera, elle, plusieurs références, sans hiérarchisation apparente mais axées sur la figure du chômeur. Ainsi seront désignés comme cible les "chômeurs en attente de versement d'indemnités ou autres prestations, (...) titulaires de l'ASS, (...) de longue durée non indemnisés”. Cette définition, bien que relativement restrictive au regard des choix effectués dans d'autres départements, s'accompagnera d'une latitude importante laissée à l'usager quant au mode d'instruction de sa demande, le choix des pièces susceptibles de justifier la légitimité de celle-ci étant largement laissé à son appréciation de même que le contenu d'une rubrique destinée à l'exposé détaillé de sa situation.

On pourrait entrer plus dans le détail des logiques qui sous-tendent les positionnements adoptés par les partenaires institutionnels locaux mais aussi des registres de justification que ces derniers mobilisent, a posteriori, pour en rendre compte. On ne s'attardera pas cependant sur les caractéristiques d'une négociation dont les termes, bien que toujours inscrits dans une histoire locale, sont largement connus : au croisement de «l'organisationnel transversal local » et de l'« administratif vertical » (Santelmann, 1995), la rencontre de stratégies institutionnelles autour d'un projet qui met en jeu l'équilibre, plus ou moins stabilisé, que les protagonistes s'efforcent de

\footnotetext{
${ }^{3}$ En premier lieu, tant sur le plan politique qu'administratif, les objectifs fixés nationalement contenaient une double orientation en apparaissant, dans le même temps, comme une réponse à des mouvements sociaux situés (manifestations de chômeurs) et comme une phase préparatoire au «volet-urgence » de la loi contre les exclusions à venir. De plus, en mettant plus l'accent sur la nécessité d'une réponse immédiate que sur le contenu de celle-ci, ils se révélaient plus volontaristes qu'explicites sur les modalités concrètes de mise en oeuvre du dispositif. Par ailleurs, les autorités départementales devaient rapporter la création du FUS aux dimensions de la revendication sociale locale, particulièrement faible dans le département, en tenant compte d'une médiatisation nationale puissante et des attentes que celle-ci était susceptible de générer. Enfin, toute décision était conditionnée par l'issue des négociations en matière de coopération institutionnelle et devait, au minimum, ne pas contredire des choix logistiques dont on a vu qu'ils furent en partie dictés par les contraintes liées au positionnement des partenaires locaux.
} 
maintenir au sein d'une configuration d'acteurs que la décentralisation a rendus plus indépendants tout en les incitant à se rapprocher.

Ce qu'on voudrait souligner ici, ce sont en premier lieu les contraintes fortes qui pesaient sur cet exercice de formalisation d'une discrimination positive largement ouverte quant aux caractéristiques des sujets visés et à sa finalité. Mais on retiendra également le caractère pragmatique d'une série de décisions qui laissent aux critères suffisamment de souplesse pour permettre, en interne, la constitution progressive d'une «jurisprudence », tout en se donnant quelques limites apparentes afin de favoriser à l'externe une préselection des candidats - par les publics eux-mêmes - et par là contenir les risques de débordement que pourrait occasionner la diffusion d'une définition semblant ne s'être dotée d'aucun critère de restriction.

\section{Les enjeux de la simplification des procédures}

Contraint de gérer le décalage entre l'étendue de l'espace d'initiative qui lui était accordé par l'administration centrale et l'étroitesse relative de la marge de maneuvre dont il disposait face à ses partenaires, l'Etat local sera bien parvenu, dans des délais trés courts, à élaborer un dispositif opérationnel. On conviendra cependant que le contenu prescriptif donné localement à cette politique publique se révèle ex post marqué par une relative indéfinition initiale. De même qu'au regard des contraintes qui pesaient sur l'opérationnalisation du dispositif, sans doute est-il plus approprié d'évoquer l'idée d'une normativité "contingente" ou "résiduelle" que de rapporter les choix effectués à l'affirmation de principes d'action pleinement assumés ${ }^{4}$.

Cependant, une chose est de s'interroger sur les conditions dans lesquelles furent prises ces décisions, une autre est d'en mesurer les effets et d'en éclairer les enjeux. Dans ce sens, deux lectures opposées peuvent être faites d'un dispositif doté d'une définition initiale "extensible" de son public, limité officiellement à deux interlocuteurs - une commission centralisée et un usager périphérique - et laissant dans une large mesure au candidat à la prestation le choix du type d'argumentation déployé pour justifier sa demande .

Selon une vision qu'on pourrait qualifier de "libérale", on mettra en avant les vertus d'un dispositif qui tend à réduire la complexité administrative, source d'inefficacité, et évite de ce fait à des usagers déjà fragilisés l'épreuve du parcours d'obstacle que leur impose trop souvent une rigueur bureaucratique marquée par un attachement excessif au respect de procédures opaques, doublée d'un cloisonnement institutionnel dont le demandeur fait seul les frais ${ }^{5}$. On verra dans le fait de ne pas avoir institué de nouveau guichet social, le moyen d'éviter la "stigmatisation" de personnes que la position d'attente passive enferme dans un statut dévalorisant. On soulignera la pertinence d'un ciblage qui, en se dispensant d'une catégorisation rigide parce qu'étanche, permet

\footnotetext{
${ }^{4}$ Pour cette raison, on s'interdira de mettre les choix effectués au seul compte de décideurs locaux tenant par là à promouvoir une certaine conception de l'action sociale. Les remarques qui suivent ne se prêtent donc à aucune conjecture quant à la «philosophie » que les représentants de telle ou telle institution locale peuvent défendre par ailleurs.

${ }^{5}$ Comme le montre Hélène Strohl, les démarches administratives à effectuer sont de manière générale d'autant plus lourdes que les usagers sont fragilisés et démunis, tout se passant comme si le poids des efforts à accomplir constituait la contrepartie de l'aide que leur accorde la collectivité (Strohl, 1988).
} 
de s'adapter à la diversité des cas individuels et, en partant des situations de fait plutôt que de catégories déjà constituées, permet de ne favoriser ni d'exclure personne a priori. Enfin, on fera remarquer qu'en laissant une marge de maneuvre aux usagers pour exprimer leur attente et argumenter leur demande au plus prés de leur vécu quotidien, tout en responsabilisant ces derniers, on se donne les moyens de tendre vers une plus grande efficacité mais aussi une plus grande équité dans la répartition d'une aide donnant à chacun ce dont il a effectivement et précisément besoin. En clair, on s'accordera sur la supériorité du principe qui régit l'aide sociale, selon lequel c'est à l'usager de prouver qu'il a bien besoin de ce qu'il demande (Joint-Lambert, Bolot-Glitter, Daniel, 1998).

A l'opposé, on trouve une logique à vocation égalitaire qui s'appuie sur la rationalité des procédures de sélection pour assurer l'égalité de tous devant l'accés aux prestations en garantissant l'automaticité de la réponse publique à ceux qui entrent de manière strictement définie dans la catégorie concernée. Selon cette logique, on verra dans la procédure retenue localement la marque du «droit précaire » qui caractérise les aides facultatives (Autès, 1999), du «droit subjectif » qui marque l'aide sociale (Alfandari, 1987) et on pointera les limites d'un dispositif qui reporte sur le public le poids de l'indétermination de sa cible en se proposant moins d'atteindre une population définie comme légitime que d'effectuer empiriquement un tri parmi les personnes qui se manifestent. On soulignera que, sous couvert d'un discours visant à responsabiliser un usager doté de «compétences sociales » et qu'il faut cesser d'infantiliser, on renoue avec les pratiques d'assistance les plus archaïques. On signalera que l'opacité de ce type de procédure est bien plus redoutable que celle qui caractérise le fonctionnement bureaucratique car elle masque des pratiques qui doivent plus au jugement de valeur qu'au diagnostic objectif et distancié. On notera enfin qu'en l'absence de critères strictement définis, l'évaluation des situations ne peut que davantage porter sur les modalités d'expression de la demande que sur la réalité des situations vécues et que l'efficacité ainsi que l'équité recherchées sont de ce fait largement illusoires, sinon suspectes lorsque ce sont les savoir-faire narratifs de l'usager, ou pire, son mérite, qui sont implicitement érigés en critères de selection.

C'est volontairement que nous opposons ici deux logiques qui sont souvent appréhendées par les gestionnaires de la protection sociale comme complémentaires. Equité dans la répartition des aides et égalité de traitement apparaissent généralement comme deux principes qu'il faut concilier tout en assurant une plus grande efficacité de la réponse publique. Les mutations de la société salariale, que traduisent la massification et la diversification des demandes d'aide financière, imposent alors de trouver un «juste équilibre » entre les deux principes, tant au niveau des grandes régulations de l'Etat-Providence qu'au niveau des organismes de protection sociale qui délivrent des prestations ${ }^{6}$. Ajoutons que, opposer terme à terme comme on le fait ici les principes de préconstruction et de construction pragmatique des catégories-cible de la

\footnotetext{
${ }^{6} \mathrm{Au}$ premier niveau, le débat s'articule autour des mécanismes d'assurance, d'assistance et de solidarité et des enjeux d'une rédéfinition socio-politique de la citoyenneté sociale (Concialdi, 1998) à l'heure d'une disjonction entre revenus du travail et prestations sociales (Mongin, 1998). Au second, dans le prolongement des travaux fondateurs de Jean-Luc Outin dévoilant l'hétérogénéité de la population concernée par les prestations financières et l'importance des interactions entre allocataires et agents d'accueil (Outin, 1979), on trouve la réflexion d'organismes sociaux qui, à l'instar de la CNAF, s'interrogent sur les conditions d'articulation entre exigence d'égalité administrative et nécessité d'une personnalisation des aides apportées (Outin,1993), en particulier face aux usagers présentant un "faible niveau d'autonomie administrative" (Caizzi, Quiroga, Riondel 1998).
} 
protection sociale ne rend que partiellement compte de la réalité. A ce titre, sans doute serait-il plus juste d'évoquer l'idée d'un continuum au sein duquel prennent place l'ensemble des prestations financières ${ }^{7}$.

Telle que nous l'avons présentée, l'opposition frontale entre les deux logiques peut donc apparaître abusivement simplificatrice. Cette présentation a cependant le mérite de rappeler que, le choix de tel ou tel mode de définition des cibles du social ne se réduit pas à une série d'exigences fonctionnelles en matière de gestion de l'attribution de prestations. Que l'aide proposée se présente ou non comme un droit exigible par le demandeur, lorsque se pose la question de la participation de l'usager à cette construction de la réponse publique, c'est bien le choix, implicite ou revendiqué, d'une conception de ce dernier en tant qu'acteur qui se trouve mis en jeu : usager irrationnel, objet de la procédure, totalement «agi » mais protégé par cette dernière versus usager acteur de la catégorisation, qui, en motivant sa demande et, dans le cas du FUS local, en étant invité à argumenter la réalité des besoins qu'il exprime, est invité à argumenter son appartenance à la catégorie concernée. Or, faut-il rappeler qu'il n'est pas indifférent, sur le plan politico-moral, de s'adresser à un usager autonome, responsable, et de le convier ainsi à faire entendre sa voix au sein d'un processus qui engage le sort qui lui sera finalement réservé ? Bien que les prises de position qui s'affirment sans nuance plus favorables à l'un ou l'autre de ces principes de sélection soient rares, les différentes versions du "subtil dosage" qui est souvent prôné masquent donc des oppositions fortes, qui mériteraient d'ailleurs à notre sens d'être plus clairement débattues. La référence à des valeurs, si ce n'est à une idéologie, n'est jamais totalement absente dés lors que se pose la question de la rationalité que l'on prête aux usagers de l'action sociale ainsi que son corollaire : la responsabilité dont on attend qu'ils fassent preuve dans le redressement de leur situation et, directement liée, celle qui peut leur être attribuée dans l'apparition de leurs problèmes.

Ces quelques remarques nous semblent nécessaires, d'abord pour insister sur le fait que les données d'enquête présentées ici n'apporteront pas un éclairage décisif à une question - celle de la légitimité qui doit être accordée au discours de l'usager - que n'épuisent pas les constats empiriques, fussent-ils apparamment concordants dans ce qu'ils mettent en evidence, et qui, plus qu'une question de recherche, demeure, et doit demeurer une question politique. Surtout, on voudrait souligner que la prise de distance que nous nous imposons à l'égard des enjeux politiques de l'action n'en n'élimine pas la portée, même si, bien sûr, nous n'avons pas ici à engager l'analyse sur ce terrain.

\footnotetext{
${ }^{7}$ Que ces dernières relèvent de droits ouverts par des cotisations, de droits fondamentaux liés à l'expression de besoins ou du devoir que s'imposent des institutions, soit respectivement de l'assurance, de l'aide sociale ou de l'action sociale (Thévenet, 1997). La variable de ce continuum peut être définie comme le degré d'ouverture d'un espace de décision destiné à la production en situation des critères d'appartenance aux catégories concernées, par des acteurs et des instances ainsi invités à prendre en compte des éléments de contexte jugés nécessaires à la juste apréciation de la situation du demandeur. La légitimité dont sont investis ces acteurs et ces instances est elle-aussi variable, mais croise généralement trois types de référence : référence à une position sur l'organigrame de l'institution ou de l'organisme qui gère le fonds ( position qui justifie par exemple la délégation de signature ) ; référence à un modèle professionnel ( le plus souvent, celui de l'assistante sociale diplômée d'Etat ); référence à une exigence de réprésentativité de l'instance de décision (c'est le principe de la commission paritaire).
} 
C'est donc modestement, mais en ayant à l'esprit que les choix que nous discutons ne sont pas neutres, qu'on interrogera une expérience de production de l'action sociale dans laquelle l'usager fut invité à se définir comme cible légitime et à argumenter cette définition de soi. En montrant comment, à partir de ce principe, se sont effectivement ajustées offre et demande de prestation, on souhaiterait à présent rendre compte d'un des effets indirects de la construction locale d'une prestation s'adressant à un usager parfaitement autonome et responsable : on veut parler de l'émergence d'un dispositif informel au sein duquel se fit officieusement et en partie la rencontre entre offre et demande de prestation, au prix d'une diversification des modalités d'expression des besoins individuels et de la réponse publique à laquelle les autorités locales n'eurent d'autre choix que de tenter de s'ajuster.

\section{L'offre effective de prestation à l'épreuve d'un dispositif informel}

Comme on l'a vu, aucune intervention extérieure ne devait officiellement s'intercaler entre un demandeur à qui il revenait d'instruire son dossier et une commission chargée de statuer sur les demandes écrites lui parvenant. L'effectivité de la procédure retenue supposait de ce fait la "transparence" ou l'"invisibilité" d'intervenants sociaux, au sens large, qui n'avaient formellement aucun rôle à jouer au sein du dispositif.

Or, l'information puis le formulaire de demande ne tardèrent pas à se diffuser officieusement en empruntant les canaux qui, au sein de configurations d'acteurs locales, relient de manière informelle les services et les personnes qui accueillent les publics en difficulté. Cette diffusion donnera lieu à des modes d'implication dans le FUS d'autant plus diversifiés qu'aucune instruction officielle ne viendra en circonscrire les modalités et ainsi réduire l'incertitude qui caractérisait la définition des publics concernés et les conditions nécessaires à l'obtention de l'aide proposée.

\section{De la mise en circulation à l'instrumentalisation de la prestation}

A l'origine des divers usages de cette prestation nouvelle par des acteurs locaux de l'aide aux publics en difficulté, on trouve des logiques institutionnelles promptes à se déployer dans l'espace laissé vacant par la relative ambiguité des énoncés politiques nationaux et la faible normativité des choix logistiques effectués localement. On trouve aussi des logiques d'acteur ayant matière à s'exercer dans le cadre de relations, ponctuelles ou suivies, entre intervenants sociaux et usagers potentiels du dispositif ${ }^{8}$.

.Des logiques institutionnelles

\footnotetext{
${ }^{8}$ Il faut ici distinguer les lieux d'accueil «traditionnels» des publics en difficulté et les lieux d'accueil que des comités de chômeurs mettront à disposition des demandeurs potentiels. Directement liée à la dimension politicomédiatique de la réponse-FUS, l'action des comités de chômeurs se déploiera sur un registre particulier, celui de la «prime» revendiquée comme un acquis social, ce qui les conduira à appuyer toute demande à de trés rares exceptions prés. L'existence de ce circuit parallèle, propre au FUS, ne fera qu'accroître la diversité des chemins empruntés par les demandeurs pour accéder à la commission d'attribution.
} 
Il serait excessif de voir derrière chaque positionnement institutionnel l'expression d'une stratégie promue par des directions tenant à tout prix à utiliser cette «opportunité » au mieux de leur intérêt. Non seulement tous les services d'accueil n'adoptèrent pas une ligne de conduite systématique à l'égard du dispositif, mais la diversité des modes d'implication doit aussi à des effets de position dans le champ de l'action sociale locale : le type de mission habituellement remplie ainsi que le degré de proximité d'avec le centre opérationnel du dispositif en rendent partiellement compte ${ }^{9}$. Il reste que pour certains organismes ou services du secteur social, l'utilisation de la marge de maneuvre disponible pouvait offrir l'occasion d'affirmer certaines prérogatives ou de consolider une position et à ce titre on ne peut isoler les logiques institutionnelles d'un contexte local de négociation permanente pour le partage des rôles en matière d'action sociale ${ }^{10}$. Au croisement de routines et de stratégies institutionnelles, on trouve des services dans lesquels des principes d'action furent clairement affirmés, d'autres qui laissèrent le soin aux intervenants sociaux de déterminer la conduite à suivre. Dans les deux cas, c'est cependant toujours dans un cadre relationnel que seront co-produites les conditions de la rencontre entre offre et demande de prestation.

\section{.Des définitions relationnelles}

Se trouvant au contact direct des personnes potentiellement concernées par la prestation, c'est aux "acteurs de terrain" que revenait la charge de donner un contenu opératoire à la doctrine de leur institution - lorsqu'il y en avait une - en contextualisant les consignes, plus ou moins précises, qui l'accompagnaient. Quelle que soit la latitude accordée à ces derniers par leur hiérarchie, c'est donc toujours dans un cadre relationnel, déterminant les conditions d'accomplissement de logiques d'acteur, que les modalités de la rencontre entre offre et demande de prestation trouveront finalement leur expression. L'éventail des pratiques fut extrêmement ouvert, parfois au sein d'un même service, certains intervenants se limitant à transmettre l'information, alors que d'autres distribuaient le formulaire et que d'autres encore aidaient à son remplissage. Dans ce dernier cas, la forme de l'aide pouvait également varier, allant de conseils succincts, au rôle "d'écrivain public", jusqu'à une instruction en tous points similaire à ce que les travailleurs sociaux ont coutume de pratiquer, c'est-à-dire signée de son auteur et où pouvait éventuellement figurer une proposition de montant. Enfin, certains intervenants s'engagèrent dans le suivi du parcours des dossiers, en contactant parfois la cellule technique de la commission d'évaluation afin de connaître l'état d'avancement d'une demande, contester une

\footnotetext{
${ }^{9} \mathrm{Si}$ la nature des missions habituellement exercées explique en partie le positionnement des organismes membres de la MUS ( ainsi le Service Social Départemental a-t-il laissé à ses assistantes sociales la possibilité d'intervenir dans le dispositif “ à titre personnel”), le mode d'implication des institutions qui n'en faisaient pas partie dépendait également de l'information dont ces dernières disposaient concernant le FUS. On peut citer le cas de ce Directeur d'association qui, estimant que "le public SDF n'était en rien concerné par le FUS", n'a pas jugé opportun de mobiliser la prestation pour cette population.

${ }^{10}$ Parmi les modes d'instrumentalisation de cette prestation apparaissant comme libre d'affectation, on trouve des stratégies déployées à l'égard d'organismes membres de la MUS (parfois partenaires au sein d'autres dispositifs) et plus globalement d'autres acteurs du champ, mais aussi à l'égard de la "clientèle". Si l'on hésite en effet à parler de "clientélisme" dans le cas de structures qui viennent en aide aux publics en difficulté, on garde à l'esprit que l'utilisation massive de la prestation pouvait constituer un moyen d'attirer ou de conserver certains publics ou encore de revendiquer le statut d'acteur localement "incontournable". On pense à ce lieu d'accueil associatif qui s'est mué en atelier d'écrivain public ouvert au tout-venant ou à ce comité de chômeurs qui tentera tout au long de l'histoire du FUS local de se placer en interlocuteur direct, en tant que "partenaire technique", de la Préfecture (sans grand succés).
} 
décision ou introduire un recours. De plus, ces pratiques se fondaient souvent sur des critères de sélection propres à chaque intervenant et qui trouvaient leur pertinence dans l'actualisation, plus ou moins stratégique mais toujours circonstanciée, de principes normatifs puisant leur origine dans des routines professionnelles, des préférences axiologiques, des positionnements à l'égard de la hiérarchie ${ }^{11}$. En clair, on retrouve là l'ensemble des processus qui, au sein de la "relation de service", conduisent des acteurs à co-produire en situation des définitions relationnelles et ainsi donner un contenu à la rencontre ${ }^{12}$.

Tout au long de l'histoire du FUS dans le département, ce dispositif informel échappera au contrôle des instances de pilotage tout en contraignant les membres de la commission à s'y ajuster en permanence. Ainsi, conscients des risques d'inégalité de traitement, ceux-ci tenteront, non sans ambiguité mais au prix d'efforts considérables, d'en compenser les effets ${ }^{13}$

\section{De la multiplicité des acteurs au partage du pouvoir normatif}

Cette diversification des modes d'accés au statut de candidat à la prestation doit beaucoup aux caractéristiques d'un dispositif qui, pour différentes raisons, a multiplié les acteurs qui pouvaient, formellement ou non, s'y estimer associés : multiplicité de partenaires officiels bien sûr, mais aussi d'associations qui, en raison du lien politique direct entre le FUS et les mouvements sociaux nationaux qui l'avaient précédé, revendiquèrent un droit de regard sur sa mise en oeuvre — on pense aux comités de chômeurs — ou qui, en vertu de leur "connaissance du terrain", s'estimèrent, plus ou moins stratégiquement, qualifiées pour contribuer à son opérationnalisation.

La coexistence de points de rencontre officiels et officieux entre offre et demande de prestation au sein du dispositif réel, réactive cependant deux interrogations. La première porte sur la capacité des partenariats locaux à garantir l'effectivité des normes d'action qu'ils se donnent, en

11 Ainsi, peut-on associer à un "habitus professionnel" les pratiques d'assistantes sociales qui ont utilisé le FUS comme une aide financière supplémentaire permettant temporairement d'élargir la gamme des outils disponibles. La relative unité des procédures et des savoir-faire correspondants n'interdisait pas cependant des variations dans les critères de sélection mobilisés, en fonction de choix puisant au registre de la compassion, motivés par la volonté de compenser les dysfonctionnements du système d'aides préexistant, ou bien stratégiquement orientés vers la direction du service. Ainsi, telle assistante sociale qui fit le choix de privilégier "les enfants qui souffrent", telle autre de privilégier les "familles qui n'ont droit à rien par ailleurs", telle autre qui se refusa à "endosser l'habit de distributeur de billets" que tend à lui imposer sa hierarchie.

${ }^{12}$ Parmi les nombreux travaux et publications portant sur l'approche microsociologique des situations d'interaction entre agent institutionnel et usager, dans lesquelles se joue en grande partie l'efficacité de l'action publique, on pourra se reporter au numéro que la revue Informations Sociales a consacré au thème de "l'accueil des publics" (Informations sociales, $n^{\circ}$ 52, 1996) ainsi qu'au numéro de la revue Recherches et prévisions consacré aux "échanges au guichet" (recherches et prévisions , $\left.n^{\circ} 46,1996\right)$.

${ }^{13}$ Face à des savoir-faire trés inégaux en matière d'argumentation de la légitimité de la demande, les membres de la commission tentèrent de ne pas désavantager les dossiers qualifiés de "maladroits", c'est-à-dire ne répondant pas aux canons du dossier de demande d'aide financière "correctement instruit". Il leur fallait dés lors tenter de resituer le dossier dans le contexte de son instruction en s'efforçant de reconstruire la "rationalité limitée" du demandeur concerné. Notons cependant que la Commission - en partie composée d'assistantes sociales - faisait preuve d'une "neutralité bienveillante" à l'égard des dossiers instruits par des travailleurs sociaux. Ce mode d'instruction constituait selon ses membres un gage de légitimité de la demande. De plus, il leur semblait nécessaire de ne pas mettre les travailleurs sociaux en difficulté dans la relation avec leur client en leur donnant le sentiment d'être déjugés. Enfin, face à la pression exercée par certains comités de chômeurs, la commission fut amenée à faire coexister cette logique d'évaluation personnalisée et la logique de «prime de noël » pour les demandeurs qui se réclamaient explicitement de cette interprétation possible de la réponse-FUS. 
tous lieux d'un dispositif et en particulier au sein de chaque organisme membre ; on est à ce titre renvoyé à un débat plus général sur l'harmonisation des normes secondaires d'adaptation des règles formelles et par là sur les conditions d'une continuité entre les logiques institutionnelles et les logiques relationnelles au sein de chaque service officiellement impliqué. La seconde interrogation concerne la place réservée aux institutions qui sont habituellement au contact des publics en difficulté, lorsque celles-ci ne sont pas associées officiellement à la mise en oeuvre d'une politique publique. On pense en particulier au monde associatif dont les compétences sont largement reconnues et utilisées par les pouvoirs publics mais dont le statut local se trouve de fait marqué par une certaine ambiguité. La question qui est posée est celle des limites officielles de tout dispositif, à travers la manière dont on trace et on garantit les frontières du territoire institutionnel de sa mise en oeuvre ${ }^{14}$. Ce n'est alors plus le contrôle du rapport qu'établissent des intermédiaires officiels entre des normes générales et des situations particulières qui est en cause mais l'appropriation informelle d'un dispositif par des instances et des acteurs qui, de fait, participent à la construction de l'offre de prestation.

Certes, des circuits informels de diffusion de l'information appuyés sur des "réseaux de sociabilité" (Parisot et Rasse, 1992) existent dans tout dispositif et si les phénomènes de "bouche à oreille", bien connus des travailleurs sociaux, permettent bien parfois à des usagers d'accéder à leurs droits, le risque existe toujours que certains soient favorisés par leur connaissance des logiques et des acteurs de l'action sociale. On sait par ailleurs que la probabilité d'accés aux prestations est directement liée à la position de l'usager au sein du dispositif d'ajustement entre offre et demande de prestation et c'est là une des origines du "non recours" qui accompagne, dans des proportions variables, toute politique publique (Van Oorschot, 1996). De plus, tout dispositif pré-suppose chez ses bénéficiaires potentiels un accés minimal à l'information ainsi qu'une capacité minimale de compréhension des démarches à entreprendre. Enfin, il semble difficile d'éviter que certains services et à l'intérieur de ces services, certains intervenants, en fonction de l'information plus ou moins complète qu'ils détiennent sur les conditions d'obtention de telle ou telle prestation, filtrent l'accés au dispositif concerné. Et ce filtrage officieux s'exercera de manière d'autant plus variable que, dans une logique de réponse sur le mode interpersonnel à l'expression de besoins individuels, l'appréciation de la demande est toujours relationnelle, contextualisée, quelle que soit l'étendue de la marge de maneuvre que permet la procédure formelle $\mathrm{e}^{15}$.

Mais ce qu'on voudrait souligner ici, c'est moins, en soi, l'existence de modes informels d'accés au dispositif ou la diversité des choix opérés par les intervenants sociaux, que la confusion qu'en l'absence de règles explicites d'attribution de l'aide, cette diversité a engendré, du côté de l'offre comme de la demande, concernant le contenu normatif de cette politique publique. Le pouvoir de désigner qui était concerné par la prestation et de définir dans quelles conditions on pouvait y

\footnotetext{
${ }^{14}$ A l'instar de la circulaire du 23 Juillet 1998 fixant les modalités de mise en oeuvre du FUS, la loi relative à la lutte contre les exclusions multiplie les appels à contribution, sans toutefois toujours préciser les limites des partenariats attendus, largement laissées à l'apréciation des autorités locales. Sur ce point voir l'article de Sylvie Hennion-Moreau (1999).

${ }^{15}$ C'est d'ailleurs l'un des problèmes que rencontrent les Commissions d'Action Sociale d'Urgence (CASU) que de simplifier les démarches nécessaires à l'obtention des prestations de secours en instaurant le principe de guichet d'accueil polyvalent, tout en limitant l'influence du point d'entrée choisi par l'usager sur l'issue de la démarche.
} 
prétendre ne fut du début à la fin ni entièrement dans les mains des demandeurs, ni tout à fait dans celles des intervenants sociaux locaux ou des membres de la commission ; il fut involontairement partagé entre les uns et les autres au travers de conjectures permanentes portant sur les logiques en jeu dans les divers lieux où, selon ces mêmes acteurs, semblait pouvoir se poser la question de la définition des problèmes que le FUS devait contribuer à régler.

En d'autres termes, l'incertitude dans laquelle furent plongés ceux qui, à un moment ou à un autre, eurent à intervenir dans le dispositif, ne fut pas limitée à une interrogation sur la cible du FUS. Elle porta, de manière tout aussi centrale, sur l'identification des acteurs qui pouvaient légitimement produire cette définition et la connaissance de leurs logiques et de leurs critères de sélection. C'est dire qu'à l'image d'un usager progressant de manière autonome au sein d'un circuit unique, capable d'un discours argumentaire dont la rationalité devait garantir la limpidité et s'adressant à une commission de ce fait parfaitement renseignée, s'est rapidement substituée celle d'une configuration d'acteurs aux contours imprécis au sein de laquelle chacun s'efforçait d'anticiper ou de reconstruire la logique d'interlocuteurs multiples.

\section{Les limites d'une analyse centrée sur la variabilité des usages de la notion d'urgence sociale}

On comprend, à la lumière de ces éléments, que le regard que portent de nombreux acteurs locaux sur les conditions dans lesquelles fut organisée la sélection des candidats au FUS soit, pour le moins, empreint d'une certaine perplexité. Et il est vrai qu'en première analyse, les données présentées ici ne plaident pas en faveur de la voie qu'on a qualifiée plus haut de "libérale" mais, bien au contraire, tendent à confirmer que cette dernière est bien celle du "droit discrétionnaire" car chacun s'y voit autorisé, de manière officieuse et dans la plus grande opacité, à faire valoir sa propre définition de la cible du dispositif. Mais cette voie apparaît aussi comme celle de l'inégalité, étant entendu qu'on ne peut garantir l'égalité devant l'accés aux prestations dés lors que coexistent pour un même dispositif, plusieurs définitions de la population visée.

Cependant, ce ne serait qu'au prix d'une certaine confusion qu'on tirerait argument de ce qui précède en faveur de la voie "égalitaire" en dénonçant — comme on l'a souvent entendu — les interprétations de la notion d'urgence qu'a occasionnées le type de dispositif mis en place localement.

En effet, une chose est de considérer que les autorités locales n'ont pas pu contrôler le déploiement de modes d'instrumentalisation de la prestation donnant lieu à autant de pratiques effectives de sélection, puis de déplorer que tous les usagers n'aient pas bénéficié, selon le point d'entrée dans le dispositif, de conditions équivalentes d'accés à la commission. Une autre est de dénoncer la multiplication de définitions considérées comme partielles, relatives, "subjectives", bref n'approchant que sous un angle particulier le contenu véritable, purement descriptif, de la notion d'urgence.

Entre les deux, il y a un pas qu'on hésite à franchir, en premier lieu car ce raccourci dans l'analyse sous-entend, en instaurant un principe d'équivalence entre objectivité des critères de sélection, égalité de traitement et justice dans la distribution, qu'il existerait une définition objective de 
l'urgence sociale et que, par suite, c'est au regard de cette définition qu'il faudrait évaluer la pertinence de tout dispositif de sélection, en ce qu'il permet ou non de se prémunir de toute interprétation erronée et arbitraire de la notion d'urgence. En d'autres termes, en opposant subjectivité des usages relationnels de la notion d'urgence et objectivité des procédures de sélection, on est amené à introduire une continuité qui nous semble abusive entre définition normative et juste définition — dans le sens de justesse et de justice — de la cible du dispositif. Mais plus encore et comme on va tenter de le montrer, on est conduit à centrer le propos sur une notion, l'urgence sociale, qui nous semble devoir être replacée au sein d'un répertoire de notions largement interchangeables en tant que catégories de discrimination positive, en ce qu'elles ont en commun de permettre d'exprimer la volonté politique d'apporter une réponse aux situations que le droit commun n'est pas en mesure de traiter.

\section{Pour une construction du sens de l'intervention auprès des publics définis comme prioritaires}

Il est vrai que si l'octroi de l'aide avait été soumis à des règles précises, l'existence d'une multiplicité de points d'entrée informels dans le dispositif n'aurait influé que sur les modes d'accés, pour les usagers potentiels, à l'information sur le FUS. Si la multiplication des intervenants officieux a favorisé une "dilution" du pouvoir légitime de définition des publics et des problèmes visés, c'est bien parce que le ciblage officiel, interne et externe, a dégagé une marge de maneuvre pour les pratiques des divers acteurs qui se sont impliqués dans le FUS.

Bien sûr, la dimension nationale du FUS, en tant que réponse à des mouvements de revendication largement médiatisés, n'est pas étrangère à cette confusion. D'aucuns y voient cependant également la marque de l'imprécision des notions mobilisées pour organiser cette sélection, en observant qu'aucune interprétation ne s'est imposée de manière univoque. Au premier rang de ces notions on trouve bien évidemment la notion d'urgence sociale.

\section{Au delà de l'urgence sociale, les limites d'une discrimination positive "objective"}

Comme le rappelle Denis Bouget ${ }^{16}$, la place de la notion d'urgence dans le dispositif peut être analysée à différents niveaux. Lorsqu'elle désigne un mode d'intervention, l'urgence introduit une référence à la temporalité de l'action publique et c'est au regard de l'exigence d'immédiateté qu'elle véhicule qu'il faut interroger un dispositif tel que le FUS, d'une part en ce qui concerne les délais effectifs de traitement des dossiers, d'autre part sous l'angle de ce que, de manière plus générale, le recours à la notion d'urgence révèle des évolutions qui traversent la gestion politique des phénomènes de pauvreté ${ }^{17}$. Mais l'urgence trouve aussi sa place dans le dispositif en tant que catégorie descriptive, mobilisée pour désigner les personnes potentiellement concernées par la prestation. Dans cette perspective, tous les rapports d'évaluation départementaux du FUS mettent en évidence le flou qui entoure la notion, dés qu'elle doit servir de support à la discrimination de cas situés. On ne s'en étonne guère lorsqu'on sait qu'au sein des services hospitaliers, les

\footnotetext{
${ }^{16}$ Voir le rapport de synthèse sur la mise en place du FUS réalisé à partir des études départementales (Bouget, 1999).

${ }^{17}$ On pourra en particulier se reporter à l'analyse que développe Zaki Laïdi (1998) de l'urgence comme "dévalorisation culturelle de l'avenir".
} 
médecins eux-mêmes sont quotidiennement en prise avec la difficulté d'objectiver une définition de l'urgence et divisés sur la question des critères légitimes correspondants (Dodier, Camus ; 1996). On ne s'étendra donc pas ici sur la multiplicité des registres de justification disponibles pour promouvoir telle ou telle définition de "l'urgence vraie" en matière d'action sociale, particulièrement quand la sélection a pour finalité l'attribution d'une prestation financière ponctuelle.

Cependant, il serait trés réducteur de s'arrêter sur cette notion et l'incertitude qui semble la caractériser. Comme on l'a vu, les différents acteurs qui ont été amenés à intervenir dans le ciblage du dispositif ont moins essayé de donner un contenu à la notion d'urgence qu'ils n'ont tenté, de manière pragmatique sinon stratégique, de saisir cette occasion d'élargir temporairement la palette des aides disponibles. Surtout, il nous semble qu'on ferait fausse route en faisant de la " vacuité du concept " qu'évoque à juste titre Christine Dourlens (1998) un trait spécifique de la notion d'urgence.

La notion de "détresse sociale", également utilisée officiellement pour désigner les personnes concernées par le FUS, n'offrait pas — et n'a pas offert - davantage de garanties d'univocité. Et au delà, peut-on penser que d'autres notions auraient pu imposer une interprétation unique?

On ne s'étendra pas ici sur les nombreux travaux qui, en "déconstruisant" les référentiels des politiques sociales, tendent à montrer que les notions mobilisées par les pouvoirs publics pour désigner les cibles de l'action sociale ou définir les finalités des actions menées n'ont d'autre pertinence que celle que les acteurs leur attribuent dans des contextes spécifiés. Par là, on fait référence, non seulement aux travaux relevant d'une micro-sociologie d'inspiration interactionniste, mais aussi à la perspective, dominante nous semble-t-il, consistant à mettre l'accent sur le caractère "socialement construit" des thématiques macro-sociales. C'est le cas pour "l'exclusion" dont les analyses ne manquent pas qui invitent les chercheurs à déplacer le regard pour interroger l'émergence et la formulation sur les différents scènes publiques de la thématique elle-même plutôt que de s'attacher à en définir les attributs positifs. Mais il n'est pas jusqu'aux notions de handicap ou de pauvreté — qui jusque là semblaient constituer les notions les plus "objectives" avec lesquelles travaillent l'aide ou l'action sociales — qui font l'objet de réflexions similaires faisant état de la multiplicité des interprétations et des usages observables et au delà, du caractère relatif de toute définition minimale ne prenant sens que dans un contexte sociohistorique déterminé ${ }^{18}$. Dans cette perspective, aucune catégorie descriptive telle que l'urgence,

\footnotetext{
${ }^{18}$ Parmi de nombreuses publications, on fait en particulier référence à Serge Ebersold (199) et Alain Giami (1995) pour ce qui concerne la notion de handicap, à Denis Bouget et Henri Noguès (1994) ainsi que Serge Paugam (1998) concernant l'exclusion, et aux débats récurrents sur la définition "objective" de la pauvreté (voir par exemple l'introduction générale de la grande enquête réalisée par le CREDOC en 1995, où la notion de pauvreté est présentée comme un "objet théorique mal défini"). De manière plus générale, outre les nombreux travaux centrés sur l'analyse de situations d'interaction entre usagers et agents institutionnels, des approches sociologiques diverses quant à leur objet où leurs orientations théoriques nous semblent converger vers un même souci de rompre avec une conception "essentialiste" des problèmes sociaux auxquels l'action sociale se donne pour objectif d'apporter une réponse, qu'il s'agisse de la perspective "constructiviste" anglo-saxonne appliquée aux "problèmes publics" (voir l'article de Christian Rinaudo, 1996), de l'analyse des politiques publiques, de type "analyse stratégique" (Meny et Thoenig) ou centrée sur les "référentiels" (Jobert et Muller), ou de la réflexion de chercheurs qui abordent la question sociale sous un angle socio-historique et montrent par là les évolutions qui traversent la formulation publique des thématiques qui y sont liées.
} 
la détresse ou le danger n'est en soi objective : c'est le lien qu'elle entretient avec ce qu'elle désigne qui apparaît ou non légitime, soit sous les traits d'une donnée "naturelle" avec la force de l'évidence, soit par la force de la loi et de la réglementation.

Or l'experience du FUS ne fait que confirmer que plus on se rapproche du terrain, c'est-à-dire de l'usager et du discours qu'il produit sur son propre parcours, plus ce lien est fragile, contestable, pour toutes les catégorisations qui n'ont d'autre support qu'un accord sémantique. Face à des cas situés, le caractère conventionnel de ce lien est toujours susceptible d'être mis à jour, en particulier par l'usager lui même ${ }^{19}$. Il faut donc convenir que ce sont sans doute moins les notions ayant une pertinence et un pouvoir d'évocation sur la scène politico-médiatique qui sont plus ou moins floues, que les situations et les parcours individuels qui se prêtent plus ou moins à une catégorisation normative subordonnée à un accord des protagonistes sur la pertinence de la définition retenue. Dés lors que les situations individuelles perdent de leur évidence, que chaque cas se vit, se raconte ou est perçu comme un cas unique, c'est donc bien le principe de discrimination positive lui-même qui pose problème, plus que le choix de telle ou telle notion pour en assurer la traduction concrète.

\section{De la multiplicité des acteurs à la mise en concurrence de multiples registres de légitimation}

Les travaux ne manquent pas qui montrent que toute évaluation de situations individuelles, familiales, professionnelles, fondée sur une classification des publics, se heurte aujourd'hui aux effets de la diversification des modèles correspondants socialement acceptables et de la massification de situations considérées jusqu'il y a peu comme atypiques. Sans doute une enquête sur les catégories mobilisées par les intervenants sociaux dans le cas du FUS confirmerait-elle la fragilité du registre intersubjectif lorsque c'est sur ce dernier que doit se fonder la pertinence d'une sélection des publics qu'il faut aider en priorité ${ }^{20}$.

On se limitera ici à observer que la légitimité conférée officiellement au discours parfaitement rationnel de l'usager fut placée en concurrence avec d'autres registres de légitimation entre lesquels les autorités locales n'ont pu ou n'ont su faire de choix : légitimité du contenu et de la

\footnotetext{
${ }^{19} \mathrm{Ne}$ serait-ce que parce que l'usager n'auto-confirme pas la définition qui lui est proposée et tente d'imposer une définition qui lui semble plus propice à l'obtention d'une réponse qu'il juge plus satisfaisante, par exemple en présentant systématiquement sa demande sous le signe de l'urgence (Cousin,1996).

${ }^{20}$ Comme le montre Christine Dourlens (op cit), si l'action sociale a toujours été le lieu d'une tension entre des catégories politico-administratives et des situations particulières, à l'heure des "normaux inutiles" (Donzelot, 1994) et des "surnuméraires" (Castel, 1995) c'est la définition du public du travail social elle-même qui semble poser problème, faute de critères stables et légitimes de mesure de l'écart à la norme, pilier traditionnel de ce repérage. Dans ce sens, c'est bien la légitimation de la ligne de partage entre clients et non clients de l'action sociale dans des contextes spécifiés qui est rendue difficile et ce, dans une certaine mesure, quel que soit le label mobilisé. Notons cependant que le refus d'un "nominalisme" excessif en la matière ne dédouane en rien les politiques de la responsabilité qui leur revient dans la détermination des objectifs poursuivis. On peut en effet se demander si, à ce niveau, l'indéfinition n'a pas parfois un caractère fonctionnel en permettant d'alimenter l'espoir d'une solution à des problèmes dont la formulation, pour donner lieu à l'affirmation d'un volontarisme politique, nécessite précisément de ne pas permettre la désignation de cas situés, à l'instar de l'usage de la notion d'insertion pour laquelle "tout se passe comme s'il fallait demeurer dans l'incertitude" (Maurel, 1994). La discontinuité entre les catégories politicomédiatiques et les catégories opératoires face à des situations singulières, sans être nécessairement recherchée, se verrait dans ce cas instrumentalisée au profit d'un discours politique soucieux de dissimuler les incertitudes et les contradictions qui entourent l'opérationnalisation de l'objectif de re-création de la cohésion sociale.
} 
forme de l'argumentaire présenté pour justifier la demande ; mais aussi légitimité de celui qui argumente, au nom d'une professionalité, de l'appartenance à une institution, du respect de la procédure, de la référence à la dimension politico-médiatique du FUS, ou d'une souffrance vécue dont on n'a que la trace écrite à travers l'expression d'une "autobiographie négative" (Trepos, 1992). De ce fait, l'incertitude sur le contenu de la norme s'est vue doublée d'une incertitude sur sa localisation officielle. Dans ces conditions, on ne s'étonnera guère que soit pointée a posteriori par de nombreux observateurs la "subjectivité" de la selection opérée par les divers intervenants sociaux. Toute lecture des situations et des comportements à visée normative, si elle est amputée de son caractère d'évidence sociale mais aussi de la légitimité des acteurs qui l'effectuent, ne peut apparaître en effet que comme une lecture "subjective".

C'est donc la mise en concurrence de différents registres de légitimation de la sélection opérée qu'il faut retenir de cette expérience et soumettre à la critique, en particulier pour ce qui nous semble en être la conséquence essentielle : cette confusion quant au contenu normatif du dispositif-FUS n'a guère permis aux acteurs de l'offre comme de la demande de prestation de se réunir autour de la construction de sens de cette réponse publique. En témoignent les propos de nombreux acteurs locaux qui évoquent l'idée d'une distribution "quasi-aléatoire", ce qu'on s'accordera à reconnaître comme le degré le plus bas de la signification, ou d'une distribution "fortement empreinte de subjectivité", ce qui enferme le sens de la démarche dans l'espace étroit de l'intime conviction - mais sans la légitimité dont est investie cette dernière en matière judiciaire - , ou, au mieux, le circonscrit aux contours de la relation interpersonnelle. $\mathrm{Au}$ croisement d'un type de procédure d'instruction des demandes et d'un mode de ciblage qui n'ont pas produit leurs propres instruments de légitimation, c'est donc bien la signification collective, donc politique au sens noble du terme, de la réponse publique qui se trouve marquée par l'indéfinition.

\section{Permettre aux acteurs de donner un sens à la réponse publique}

On voit que, face à un ensemble de notions (l'urgence, la détresse, etc.) qu'on s'accordera à reconnaître comme largement interchangeables et sauf à renoncer à aider ceux qui en ont le plus besoin, la question qui se pose est bien, lorsqu'est en jeu la définition des publics prioritaires de l'action sociale, de faire le choix d'une logique de sélection et, partant, d'une logique de justification de cette sélection. Or celle-ci sera toujours contestable, ne serait-ce que par une sociologie critique dévoilant le travail de construction des thématiques sociales, si ce n'est par les publics eux-mêmes sur le registre de l'intersubjectivité. Comment dés lors donner un sens à cette opération forcément imparfaite et d'une certaine manière, forcément injuste, si l'on ne se résout pas à fonder la justice sur le seul respect des procédures ${ }^{21}$ ?

Une première solution consiste à faire en sorte que les publics ciblés se désignent en partie euxmêmes, ce qui revient à reporter l'incertitude sur l'usager en faisant l'hypothèse implicite que les personnes concernées...se reconnaitront. On en mesure les conséquences : faire l'hypothèse de la

\footnotetext{
${ }^{21}$ On fait référence à la distinction qu'opère Michel Autès entre "la justice sociale conçue comme découlant de la déclinaison d'une conception du Bien " et la justice sociale " comme résultant de l'application conforme des règles" (op cit p 103).
} 
rationalité de l'usager-acteur c'est aussi faire une hypothèse sur le "système" dans lequel cette rationalité est appelée à s'exercer. Dés lors, une prestation qui vise officiellement un usager parfaitement autonome s'adressera en fait à des usagers ainsi qu'à une multiplicité d'intervenants dotés d'une rationalité limitée par leurs savoir-faire et leur position dans le dispositif réel, au prix d'une mise en concurrence de divers registres de légitimation de la définition proposée et finalement de l'impossibilité pour les acteurs concernés de donner une signification collective à la démarche de construction des catégories-cibles.

On peut également se doter de critères stricts produisant une norme clairement repérable, si ce n'est dans son contenu, au moins dans sa localisation. Comme on l'a vu, on court alors le risque de figer des catégories qui ne permettront que difficilement de répondre aux exigences de souplesse et d'individualisation de l'aide, n'offriront guère de garanties d'un traitement rapide des demandes et connaîtront inévitablement les effets de seuil qui marquent les prestations soumises à ce type de critères. Plus fondamentalement, l'espace de légitimité de la catégorisation étant ici clairement circonscrit à celui de la procédure, s'avance dés lors le spectre d'un "droit des exclus" - si l'on est dans une logique de droit, on passe d'un droit à l'assistance à un droit de l'assistance - doté d'une définition légale des ayant-droit de la solidarité qui ne laisse de place pour en construire la signification ni à un usager irrationnel et simple objet d'une procédure, ni à un intervenant social réduit au rang de vérificateur de la conformité de la démarche.

Une troisième solution passe par la légitimation d'une catégorie d'acteurs pour adapter des principes généraux à des situations particulières - quitte à s'en remettre à leur «intime conviction »- en s'appuyant sur ce qui a fait défaut au FUS local : la construction de la compétence professionnelle conjointement à la construction des publics et des problèmes qu'ils rencontrent, de manière à donner un sens à la seconde parce que la première est institutionnellement légitimée. C'est là sans doute la seule voie qui permette d'associer l'usager à la production du sens de la réponse publique, en assurant une présence de la collectivité dans l'espace singulier de la relation interpersonnelle. Mais deux conditions doivent pour cela être remplies : tout d'abord se donner les moyens de doter le travail social du monopole de ce qui devient un «diagnostic social»; ensuite, ne pas systématiquement pointer la subjectivité des critères qu'utilisent les travailleurs sociaux dés qu'on observe de prés leur manière de travailler. Cela implique sur le premier point d'être attentif aux conditions de ce choix à l'épreuve des partenariats locaux ${ }^{22}$, sur le second d'être au fait des enjeux qui entourent ce même choix à la lumière de ce que la sociologie nous apprend du recours public aux "professions" 23 .

\footnotetext{
${ }^{22}$ L'expérience du FUS confirme en effet, s'il en était besoin, le poids des enjeux stratégiques qui entourent la mise en place des partenariats institutionnels dans le prolongement de transferts de compétences issus de la décentralisation. Or une des conséquences de cette négociation permanente est de placer différents modèles professionnels en compétition (Chopart, 1994).

${ }^{23}$ On fait référence aux enjeux de la construction de l'adéquation entre la formulation des objectifs que se donnent les politiques publiques et l'identification des compétences disponibles sur le "marché des qualifications ».' L'exigence de rationalité administrative dans la mise en oeuvre de la réponse publique et la volonté des groupes professionnels de se voir reconnaître le statut d'expert se rencontrent généralement sur la nécessité de manifester l'objectivité et la neutralité des savoirs mobilisés. Or, si le travail social se voit si souvent opposer la subjectivité de ses diagnostics, c'est dans une large mesure parce qu'il n'est que partiellement parvenu à asseoir sa position au sein de cette transaction, en particulier parce que lui fait défaut la possibilité de se prévaloir de la maîtrise d'un savoir scientifique spécifique (Ion et Tricart, 1992). De ce fait, entre un travailleur social magistrat et un travailleur social ingénieur, sans doute y-a-t-il un équilibre à trouver qui contienne la tentation corporatiste et ses effets pervers : le
} 
Certes, recourir au travail social ne supprime pas la nécessité d'un questionnement sur les moyens à dégager afin d'apporter une réponse structurelle à des situations de précarité qu'on sait chroniques. De même que ne saurait par là être gommé le caractère limité de prestations uniquement financières lorsque ce sont les liens qui unissent les individus à la collectivité qui font défaut. Ce qui est ici en jeu, c'est seulement — mais est-ce négligeable ? - de donner un sens à l'exercice d'une des formes de la solidarité, au demeurant nécessaire, mais que guette toujours le risque de voir par là acheté le silence des plus démunis.

Il n'est pas inutile à ce titre de garder à l'esprit que c'est aussi et avant tout un choix politique que d'attendre de l'action sociale qu'elle soit au service d'un usager capable de faire entendre sa voix dans les interstices d'un droit protecteur.

\section{BIBLIOGRAPHIE}

Alfandari E., Aide sociale et action sociale, Paris, Dalloz, 1987.

Autès M., Les territoires politiques de l'action sociale, Informations sociales, nº76, 1999.

Bouget D. et Noguès H., Evaluation des politiques de lutte contre les exclusions sociales, Revue Française des Affaires Sociales (RFAS), n² 2, avril-juin, 1994.

Bouget D., Mieux connaître les publics, les problèmes et les pratiques révélés par la mise en place des fonds d'urgence sociale en France, rapport au Ministère de 1'Emploi et de la Solidarité, Direction de l'Action Sociale, 1999.

Caizzi A., Quiroga A., Riondel J.F., Une enquête sur les usagers des CAF dits « à faible niveau d'autonomie administrative », Recherches et Prévisions, n50-51, 1998.

Castel R., Les métamorphoses de la question sociale, Fayard, 1995.

Chopart J.N., Des professions en crise, Informations Sociales, n³8, 1994.

Concialdi P., Entre l'assistance et l'assurance, la solidarité, Sociétés et Représentations, décembre 1997.

Cousin O., Les mutations du travail social : de la transformation du public aux changements dans les modes de prise en charge, Sociologie du travail, n², 1996.

Dodier N. et Camus A., Les urgences à l’hôpital, Informations Sociales, n52, 1996.

Donzelot J., L'Etat-animateur, Esprit 1994.

Dourlens C., Dans les labyrinthes de l'urgence. Le cas de l'administration sanitaire et sociale, CERPE, mai 1998.

Ebersold S., L'aggiornamento du champ du handicap, Regards sociologiques, 1995.

Giami A., Problèmes de santé et/ou de société, Informations Sociales, n²42, 1995.

Hennion-Moreau S., La mobilisation des institutions sociales, Revue de Droit Sanitaire et Social, avril-juin 1999.

Ion J. et Tricart J.P., Les travailleurs sociaux, La Découverte, Paris 1992.

pouvoir discrétionnaire d'un côté, c'est-à-dire l'auto-légitimation systématique, la dissociation entre un «social de gestion » et un « social d'intervention » de l'autre, c'est-à-dire le risque de voir les professionels s'éloigner du terrain à mesure qu'ils grimpent dans l'échelle des qualifications. 
Joint-Lambert M.T., Bolot-Glitter A., Daniel C., Les politiques sociales, presses de la FNSP, Dalloz, 1997.

Laïdi Z., L'urgence ou la dévalorisation culturelle de l'avenir, Esprit, février 1998.

Maurel E., Le statut de l'insertion, Informations Sociales, n³8, 1994.

Mongin O., Le nouveau partage des rôles entre l'assurance et la solidarité, Esprit, mars-avril 1998.

Outin J.L., Familles et droits sociaux : les modèles d'accés aux prestations familiales, Ed CNAF, coll Etudes CAF, Paris, 1979.

Outin J.L., La modernisation des caisses d'allocations familiales face à la diversité des allocataires, $R F A S \mathrm{n}^{\circ} 3$, juillet-septembre 1993.

Paugam S., L'exclusion: généalogie d'un paradigme social, Sociétés et Représentations, décembre 1997.

Parisot D. et Rasse P., Les pauvres face à leur administration : communication et accés au droit familial, $R F A S \mathrm{n}^{\circ} 2$, avril-juin 1992.

Rinaudo C., Qu'est-ce-qu'un problème social? Les apports théoriques de la sociologie anglosaxonne, Cahiers du SOLIIS, n²1, juin 1995.

Santelmann P., Lutte contre les exclusions. Sortir des logiques de cloisonnement, RFAS n²-3, avril-septembre 1995.

Strohl H., Les exclus et les organismes sociaux, Revue Française d'Administration Publique, $\mathrm{n}^{\circ} 45$, janvier-mars 1988.

Thévenet A., L'aide sociale aujourd'hui aprés la décentralisation, ESF éditeurs, 1997.

Trépos J.Y., Sociologie de la compétence professionnelle, Presses Universitaires de Nancy, 1992.

Van Oorschot W., Les causes du non recours, Recherches et Prévisions n43, mars 1996. 